Article

\title{
X-ray Flux and Spectral Variability of Blazar H 2356-309
}

\author{
Kiran A. Wani and Haritma Gaur* \\ Aryabhatta Research Institute of Observational Sciences (ARIES), Manora Peak, Nainital 263002, India; \\ kiiran.wani2@gmail.com \\ * Correspondence: harry.gaur31@gmail.com
}

Received: 6 July 2020; Accepted: 31 July 2020; Published: 10 August 2020

\begin{abstract}
We present the results of timing and spectral analysis of the blazar H 2356-309 using XMM-Newton observations. This blazar is observed during 13 June 2005-24 December 2013 in total nine observations. Five of the observations show moderate flux variability with amplitude $1.7-2.2 \%$. We search for the intra-day variability timescales in these five light curves, but did not find in any of them. The fractional variability amplitude is generally lower in the soft bands than in the hard bands, which is attributed to the energy dependent synchrotron emission. Using the hardness ratio analysis, we search for the $X$-ray spectral variability along with flux variability in this source. However, we did not find any significant spectral variability on intra-day timescales. We also investigate the X-ray spectral curvature of blazar $\mathrm{H} 2356-309$ and found that six of our observations are well described by the $\log$ parabolic model with $\alpha=1.99-2.15$ and $\beta=0.03-0.18$. Three of our observations are well described by power law model. The break energy of the X-ray spectra varies between 1.97-2.31 keV. We investigate the correlation between various parameters that are derived from log parabolic model and their implications are discussed.
\end{abstract}

Keywords: BL Lacertae objects; general—BL Lacertae objects; individual; H 2356-309—galaxies; active

\section{Introduction}

Blazars are highly luminous AGNs (Active Galactic Nuclei) that emit in all accessible wavelengths ranging from radio to high energy gamma rays. BL Lacertae objects (BL Lacs) and flat spectrum radio quasars (FSRQs) collectively belongs to Blazars. Blazars show very strong continuum with featurless optical spectrum, which is thought to be due to relativistic jets pointing nearly to our line of sight $\left(\leq 10^{\circ}\right)$ [1]. Blazar emission is mostly non-thermal in nature, shows variable polarization, and emitted continuous Doppler boosted spectra.

The broadband spectral energy distribution of blazar is characterized by a double peaked structure. The first hump at lower energies is attribued to the synchrotron emission from relativistic electrons in a jet, while the high frequency hump is thought to be produced by inverse Compton scattering from the same electron population with the synchrotron photons (SSC, Synchrotron Self Compton models; e.g., [2]) or with external ambient photons originated in the BLR (Broad Line region), torus (EC, External Compton models; e.g., [3]). The peak of the low-energy spectral component is found at X-ray/UV energies for high energy peaked blazars (HBLs/HSPs) and at optical/IR (infrared) energies for low-energy peaked blazars (LBLs/LSPs). The peak of the high energy spectral component for HSPs occurs at $\mathrm{GeV} / \mathrm{TeV}$ energies, while, for LSPs, it is usually at MeV/ GeV energies ([4,5]).

Blazars are highly variable on different timescales across the whole electromagnetic spectrum [6]. If the variability time is less than a day then it is referred to as intra-day variability (IDV) [7] and variations over a few days to months is known as short term variability (STV). When the variations range from several months to years or even decades, then it is termed as long term variability (LTV) [8]. 
HSP blazars are brightest in X-ray bands and show strong flux variability over diverse time-scales ranging between minutes to years (e.g., [9-14]).

The blazar H 2356-309 is hosted by an elliptical galaxy [15] located at a redshift $z=0.165$ $( \pm 0.002)[16]$. H 2356-309 is classified as a high frequency peaked blazar as the X-ray spectrum of this blazar was characterized by a broken power law with a synchrotron peak lying at $1.8 \mathrm{keV}$ using BeppoSAX observations $[17,18]$. It was discovered in the VHE regime by the H.E.S.S. Cherenkov telescope during June to December 2004 [19]. At X-ray energies, H 2356-309 was initially detected by the UHURU satellite [20] and subsequently by the HEAO-I satellite [21]. It has been observed with many observing facilities, like BeppoSAX [17], NuSTAR [22], and Cosmic Origin Spectrograph (COS), on board the Hubble Space Telescope (HST) [23].

Multi-wavelength observations of H 2356-309 with RXTE Satellite, HESS, ROTSE-IIIc, Nancay radio telescope, XMM-Newton \& ATOM telescope [24] suggested that the broadband spectral energy distribution (SED) can be simply fitted with a synchrotron self-Compton (SSC) model with a double-peak structure, in which the peaks in X-ray and VHE are produced via synchrotron radiation and inverse Compton scattering, respectively [24]. H 2356-309 was observed with NuSTAR in a time exposure of $21.90 \mathrm{ks}$ on 18 May 2016 [22]. However, they did not find any significant intra-day variability or spectral variation in their observations.

In the current study, we present XMM-Newton observations of blazar H 2356-309 held in between June 2005-December 2013. Our aim is to study their flux and spectral variability in the 0.3-10 keV energy range. Additionally, we fit the spectra of $\mathrm{H}$ 2356-309 using various models to study their X-ray spectral curvature and to constrain its break energy. The observing log of the XMM-Newton data for H 2356-309 blazar is given in Table 1.

Table 1. Observation Log of XMM-Newton data for Blazar H 2356-309.

\begin{tabular}{cccc}
\hline $\begin{array}{c}\text { Obs. Date } \\
\text { (yyyy-mm-dd) }\end{array}$ & Observation ID & $\begin{array}{c}\text { Total Elapsed } \\
\text { Time (ks) }\end{array}$ & $\begin{array}{c}\text { Exposure Time }{ }^{2} \\
(\mathbf{k s})\end{array}$ \\
\hline $2005-06-13$ & 0304080501 & 16.75 & 16.52 \\
$2005-06-15$ & 0304080601 & 17.04 & 16.62 \\
$2007-06-02$ & 0504370701 & 129.59 & 109.93 \\
$2012-11-18$ & 0693500101 & 118.08 & 80.25 \\
$2013-12-02$ & 0722860101 & 22.19 & 14.86 \\
$2013-12-03$ & 0722860701 & 63.48 & 44.50 \\
$2013-12-10$ & 0722860201 & 105.48 & 73.05 \\
$2013-12-12$ & 0722860301 & 107.75 & 74.99 \\
$2013-12-24$ & 0722860401 & 99.03 & 69.41 \\
\hline
\end{tabular}

${ }^{1}$ Full time interval for the exposure; ${ }^{2}$ Weighted live time of CCDs in the extraction region.

\section{XMM-Newton Observations and Data Analysis}

Blazar H 2356-309 is observed by the European Photon Imaging Camera (EPIC) on board the XMM-Newton satellite [25]. The EPIC instrument provides imaging and spectroscopy in the energy range from 0.2 to $15 \mathrm{keV}$ with a good angular resolution (PSF $=6$ arcsec FWHM) and a moderate spectral resolution $(\mathrm{E} / \Delta \mathrm{E} \sim 20-50)$. In this work, we have only considered the EPIC-pn data, as it is most sensitive and less affected by the photon pile-up effects.

Data reduction is performed with the use of XMM-Newton Science Analysis System (SAS) ${ }^{1}$ version 18.0.0 for the LC extraction. The XMM-Newton EPCHAIN pipeline is used to generate the event files. We extracted the high energy $(10 \mathrm{keV}<\mathrm{E}<12 \mathrm{keV})$ light curve for the full frame of the exposed CCD in order to identify flaring particle background. We did not find any significant background flares in our observations. We restrict our analysis to the $0.3-10 \mathrm{keV}$ energy range, as data

1 https://www.cosmos.esa.int/web/xmm-newton/sas. 
below $0.3 \mathrm{keV}$ are considerably contaminated by noise events and data above $10 \mathrm{keV}$ are usually dominated by background flares. From total nine observation data sets, three frames are in Timing mode and six are in Imaging mode.

In imaging mode, source region is extracted using a circle of 50 arcsec radius centered on the source. Background light curve is obtained from the region that corresponds to circular annulus centered on the source with inner and outer radius of 62.5 arcsec and 125 arcsec, respectively. In timing mode, the source region is extracted using a rectangular box of 20 pixels along RAWX centered on source verticle strip. The background light curve is obtained from the source free region of 20 pixels along RAWX. Pile up effects are examined for each observation by using the SAS task EPATPLOT and we found that mostly triple and quadruple events are affected by the pile-up effects; hence, we only extracted the single and double events for our observations. We did not find pile up in our observations. Finally, we obtained source LCs for the $0.3-10 \mathrm{keV}$ band (corrected for background flux and given in unit of counts s${ }^{-1}$ ), sampled evenly with a fixed bin size of $\Delta t=0.5 \mathrm{ks}$. Redistribution matrices and ancillary response files were produced using the SAS tasks rmfgen and arfgen. The pn spectra $(0.6-10 \mathrm{keV})$ were created by the SAS tool XMMSELECT and grouped to have at least 30 counts in each energy bin in order to ensure the validity of $\chi^{2}$ statistics.

\section{Analysis Techniques}

\subsection{Excess Variance}

Blazars show rapid and strong flux variations on diverse timescales across the EM spectrum. To quantify the strength of the variability, excess variance $\sigma_{X S}[26,27]$ and fractional rms variability amplitude $F_{v a r}$ are often calculated. Excess variance is a measure of source intrinsic variance determined by substracting the variance that arises from measurement errors from the total variance of the observed LC. If a LC consisting of N measured flux values $x_{i}$ with corresponding finite uncertainties $\sigma_{e r r, i}$ arising from measurement errors, then the excess variance is calculated as follows:

$$
\sigma_{X S}^{2}=S^{2}-\bar{\sigma}_{e r r}^{2}
$$

where $\bar{\sigma}_{\text {err }}^{2}$ is the mean square error, defined as

$$
\bar{\sigma}_{e r r}^{2}=\frac{1}{N} \sum_{i} \sigma_{e r r, i}^{2}
$$

and $S^{2}$ is the sample variance of the LC, as given by

$$
S^{2}=\frac{1}{N-1} \sum_{i}\left(x_{i}-\bar{x}\right)^{2}
$$

where $\bar{x}$ is the arithmatic mean of $x_{i}$

The normalized excess variance is $\sigma_{N X S}^{2}=\frac{\sigma_{X S}^{2}}{\bar{x}^{2}}$ and the fractional rms variability amplitude, $F_{v a r}[28,29]$, which is the square root of $\sigma_{N X S}^{2}$ is thus

$$
F_{v a r}=\sqrt{\frac{S^{2}-\bar{\sigma}_{e r r}^{2}}{\bar{x}^{2}}}
$$

The uncertainty on $F_{v a r}$ is given by [30].

$$
\operatorname{err}\left(F_{\text {var }}\right)=\sqrt{\left(\sqrt{\frac{1}{2 N}} \frac{\bar{\sigma}_{\text {err }}^{2}}{\bar{x}^{2} F_{v a r}}\right)^{2}+\left(\sqrt{\frac{\bar{\sigma}_{e r r}^{2}}{N}} \frac{1}{\bar{x}^{2}}\right)^{2}}
$$


In Table 2, dashes indicate that the sample variances were smaller than the mean square errors, so that no fractional variance could be claimed. When $F_{v a r}>3 \operatorname{err}\left(F_{v a r}\right)$, then only we consider a strong evidence for variability to be present.

Table 2. X-ray variability parameter $F_{\text {var }}$.

\begin{tabular}{ccccc}
\hline $\begin{array}{c}\text { Obs. Date } \\
\text { (yyyy-mm-dd) }\end{array}$ & Observation ID & $\begin{array}{c}\boldsymbol{F}_{\text {var }} \mathbf{( \% )} \\
\text { Soft (0.3-2 keV) }\end{array}$ & $\begin{array}{c}\boldsymbol{F}_{\text {var }} \mathbf{( \% )} \\
\text { Hard (2-10 keV) }\end{array}$ & $\begin{array}{c}\boldsymbol{F}_{\text {var }} \mathbf{( \% )} \\
\text { Total (0.3-10 keV) }\end{array}$ \\
\hline $2005-06-13$ & 0304080501 & - & - & - \\
$2005-06-15$ & 0304080601 & $0.49 \pm 0.84$ & - & $0.42 \pm 0.85$ \\
$2007-06-02$ & 0504370701 & $1.62 \pm 0.12$ & $4.82 \pm 0.35$ & $1.73 \pm 0.11$ \\
$2012-11-18$ & 0693500101 & $2.28 \pm 0.12$ & $4.8 \pm 0.30$ & $2.69 \pm 0.11$ \\
$2013-12-02$ & 0722860101 & $0.67 \pm 0.69$ & - & - \\
$2013-12-03$ & 0722860701 & $0.64 \pm 0.43$ & $1.78 \pm 1.16$ & $0.72 \pm 0.35$ \\
$2013-12-10$ & 0722860201 & $1.63 \pm 0.17$ & $1.59 \pm 1.09$ & $1.71 \pm 0.16$ \\
$2013-12-12$ & 0722860301 & $1.65 \pm 0.17$ & $2.01 \pm 0.85$ & $1.77 \pm 0.15$ \\
$2013-12-24$ & 0722860401 & $2.02 \pm 0.15$ & $3.12 \pm 0.52$ & $2.25 \pm 0.14$ \\
\hline
\end{tabular}

\subsection{Hardness Ratio}

To search for spectral changes over a broad X-ray band, hardness ratio (HR) is normally used (e.g., [31,32]). We extracted LCs in two energy bands, here defining $(0.3-2 \mathrm{keV})$ as the soft band and $(2-10 \mathrm{keV})$ as the hard band. We then computed hardness ratio, which is defined as follows:

$$
H R=\frac{(H-S)}{(H+S)}
$$

and the error in $H R\left(\sigma_{H R}\right)$ is calculated, as follows:

$$
\sigma_{H R}=\frac{2}{(H+S)^{2}} \sqrt{S^{2} \sigma_{H}^{2}+H^{2} \sigma_{S}^{2}}
$$

where $S$ and $H$ are the net count rates in the soft $(0.3-2 \mathrm{keV})$ and hard $(2-10 \mathrm{keV})$ bands, respectively, while $\sigma_{S}$ and $\sigma_{H}$ are their respective errors.

\subsection{Spectral Analysis}

The XSPEC software package version $12.10 .1 \mathrm{f}$ is used for spectral fitting. The Galactic absorption $n_{H}$ is fixed to be $1.3 \times 10^{20} \mathrm{~cm}^{-2}$ [33] and the Xspec routine "cflux" is used to obtain unabsorbed flux and its error.

As we know that X-ray spectra of blazars are well described by a single power-law or a broken power law. References [34,35] found that there is curvature in the blazar spectra which arise due to log parabolic electron distributions. Hence, they are also well described by the log parabola model (e.g., [36,37]). We fit each spectra using three models which are defined as follows:

1. Power law model, which is defined by $k E^{\Gamma}$. It is characterized by photon index $\Gamma$, redshift $z$, and Normalization $k$.

2. Logarithmic parabola model which is defined by $k E^{\left(-\alpha+\beta \log \left(E / E_{1}\right)\right.}$ (e.g., $\left.[34,35,38]\right)$. The lowest probed energy $E_{1}=0.6 \mathrm{keV}$ is fixed. It is characterized by three parameters, local spectral index $\alpha$, curvature parameter $\beta$, and normalization $k$.

3. Broken power law model is defined by $k E^{\Gamma_{1}}$ for $E<E_{\text {break }}$ and $k E^{\Gamma_{2}}$ otherwise. This model is used to infer the break energy in those observations which are well fitted by log parabola model. It is characterized by spectral indices $\Gamma_{1}, \Gamma_{2}$, break energy $E_{b r e a k}$, redshift, and normalization $k$. 


\section{Results}

\subsection{Flux and Spectral Variability}

We analysed nine observations of the blazar H 2356-309 using XMM-Newton satellite data (downloaded from HEASARC Data archive ${ }^{2}$ ). All obtained light curves are shown in Figure 1. We calculated fractional rms variability amplitude $F_{\text {var }}$ in various energy range, i.e., $0.3-10 \mathrm{keV}$ (total), 0.3-2 keV (soft band), and 2-10 keV (Hard band) in order to search for the flux variability in these nine observations. For all LCs X-ray variability parameter $F_{v a r}$ is presented in Table 2 . It can be seen that the flux variability is observed in five LCs out of total nine LCs. Additionally, we observed flux variability in soft band (0.3-2 keV) for all of those five LCs. However, in hard band (2-10 keV), out of those five LCs we oserved significant flux variations only in three LCs.
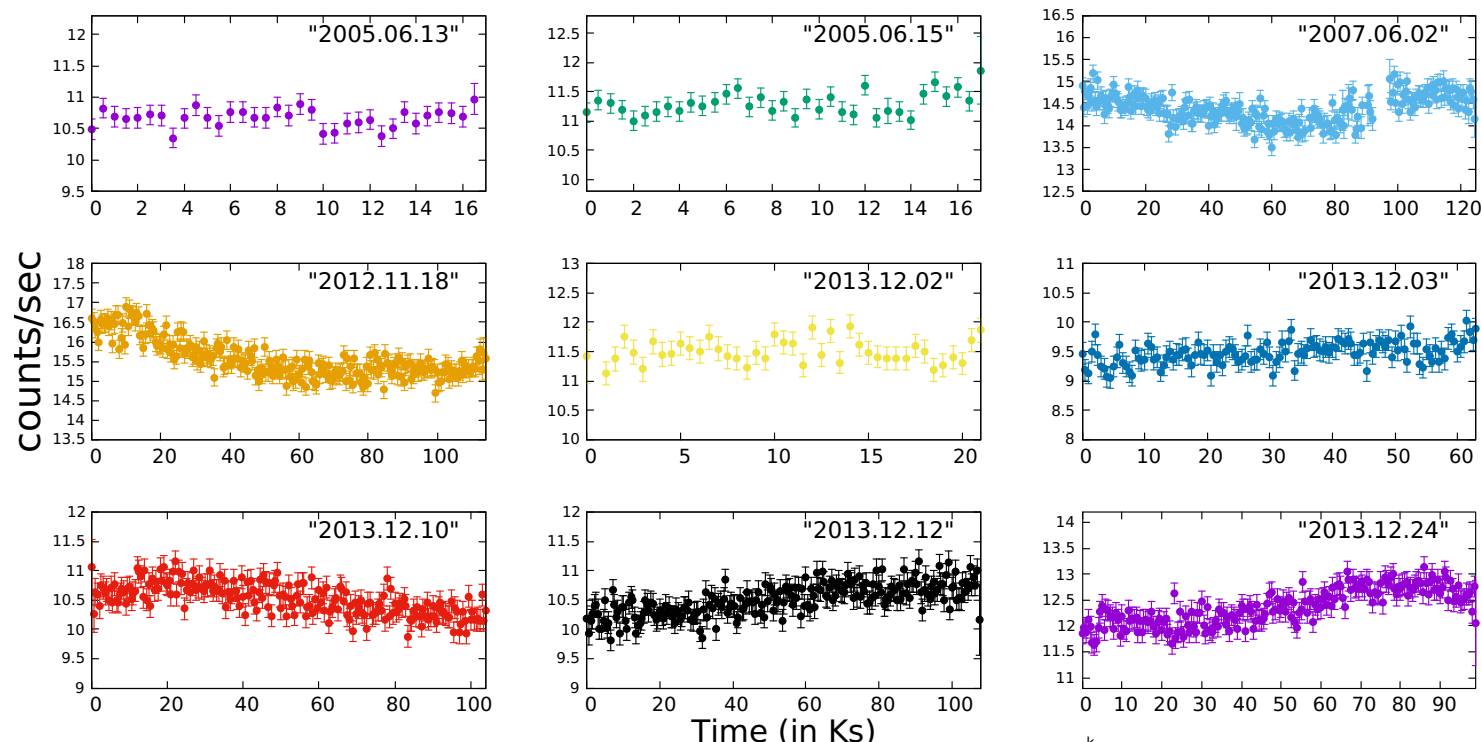

Figure 1. Light curves of H 2356-309 of XMM-Newton Observations.

To search for spectral variability along with flux variability, hardness ratio (HR) is computed. Figure 2 shows the hardness ratio for all of the observations. We did not find any significant spectral change which is obvious from the HR plots. Quantitative variations in HR using a standard $\chi^{2}$ test is obtained, as follows,

$$
\chi^{2}=\sum_{i} \frac{\left(x_{i}-\bar{x}\right)^{2}}{\sigma_{i}^{2}}
$$

where $x_{i}$ is the HR value, $\sigma_{i}$ is its corresponding error, and $\bar{x}$ is the mean HR value. We only considered a variation in the HR to be significant if $\chi^{2}>\chi_{0.90, v}^{2}$, where $v$ is the number of degrees of freedom (DoF) and the significance level is set to 0.90 . These results are provided in Table 3 , where we see that for each source $\chi^{2}<\chi_{0.90, v}^{2}$, hence no significant spectral variations are detected in any observation.

2 https://heasarc.gsfc.nasa.gov/docs/archive.html. 

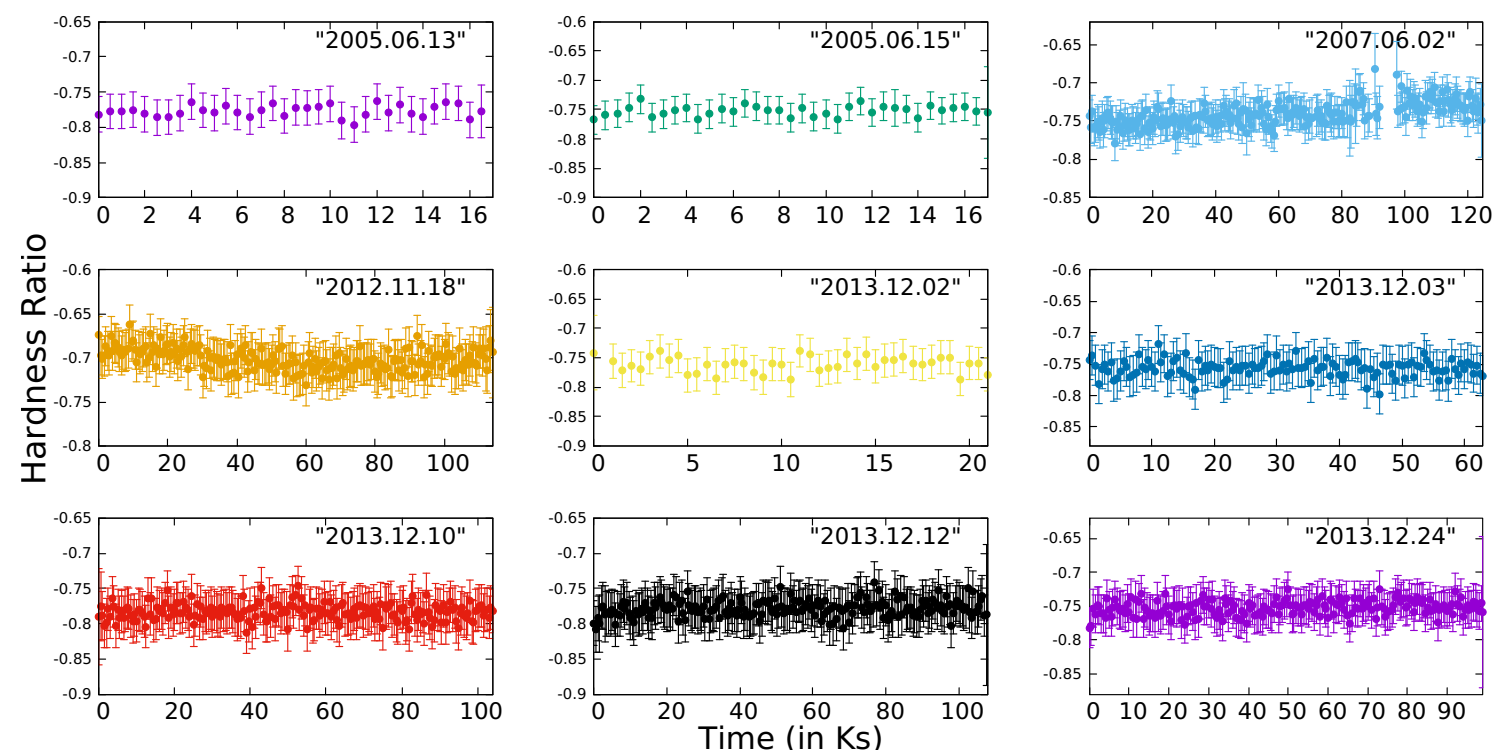

Figure 2. Hardness Ratio of H 2356-309.

Table 3. Temporal Variation of hardness ratio (HR) of H-2356 309.

\begin{tabular}{cccc}
\hline $\begin{array}{c}\text { Obs. Date } \\
\text { (yyyy-mm-dd) }\end{array}$ & DoF & $\chi^{\mathbf{2}}$ & $\chi_{\mathbf{0 . 9 0}}^{\mathbf{2}}$ \\
\hline $2005-06-13$ & 33 & 3.63 & 43.745 \\
$2005-06-15$ & 34 & 4.56 & 44.903 \\
$2007-06-02$ & 211 & 99.13 & 237.717 \\
$2012-11-18$ & 225 & 64.10 & 252.578 \\
$2013-12-02$ & 33 & 9.21 & 43.745 \\
$2013-12-03$ & 124 & 21.61 & 144.562 \\
$2013-12-10$ & 207 & 29.30 & 233.466 \\
$2013-12-12$ & 207 & 30.31 & 233.466 \\
$2013-12-24$ & 198 & 31.41 & 223.892 \\
\hline \multicolumn{4}{c}{ Bin size $=0.5 \mathrm{ks}}$.
\end{tabular}

\subsection{Spectral Fitting Results}

We fit all nine observations using power law (PL), log parabolic (LP), and broken power law model (BKN), and the results of spectral fitting are provided in Table 4. To choose the best spectral model between PL and LP model, we performed the F-test using the values of $\chi^{2}$ and degree of freedom (dof) for both of the models. LP model provides a better fit than the PL model if the value of F-statistic $>1$ and the corresponding null hypothesis probability, $p<0.01$. It can be seen from the F-test results that six out of nine observations of the blazar are well described by the LP model, where $\alpha$ varies between $1.99-2.15$ and curvature $\beta$ varies between $0.03-0.18$. Three observations of $H$ 2356-309 are well described by the PL model, as can be seen from the high values $(p>0.01)$ of null hypothesis probability. In these observations, $\alpha$ varies between 2.16-2.28. The observations that are well fitted by $\log$ parabolic model are also fitted by broken power law model to constrain the break energy $E_{b}$. It is found that the break energy varies between $1.97-2.31 \mathrm{keV}$ during our observations. The model-fitted spectra and the data-to-model ratios for each observation are plotted in Figure 3. 

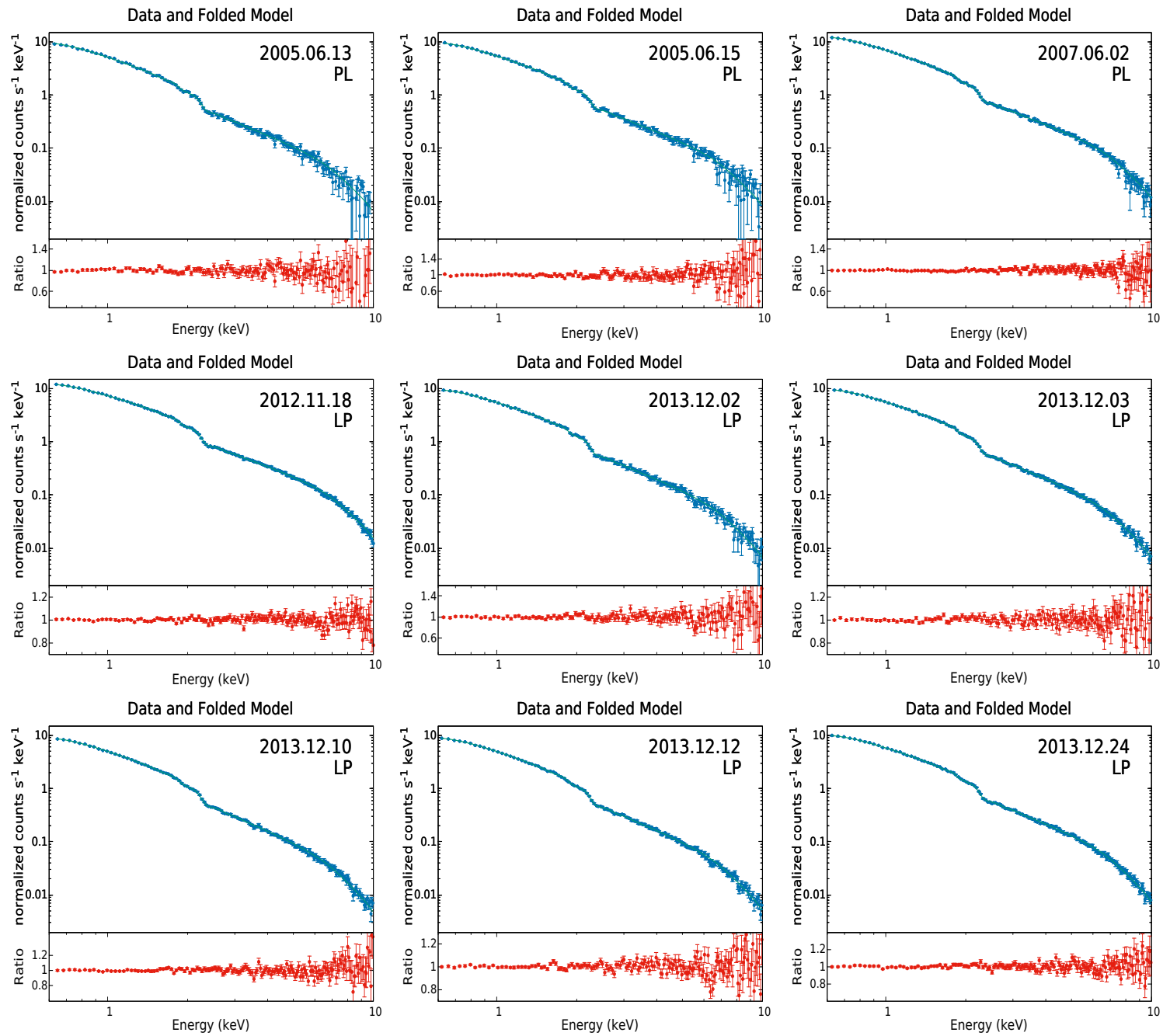

Figure 3. The spectra and ratio (data/model) of best fitted model for XMM-Newton observations of H 2356-309.

\subsection{Correlation betweeen Various Parameters}

We investigated the correlation between various parameters of LP model i.e., $\alpha, \beta$, flux, and $F_{v a r}$, and all are shown in Figure 4. We derived the Spearman correlation coefficient $(\rho)$ between $F_{v a r}$ versus flux in order to check whether variability amplitude is correlated with flux. We found $\rho=0.5$ with null hypothesis probability $p=0.17$ between them. Hence, we can conclude that there is no significant correlation between them. Additionally, we investigated the correlation between spectral index $\alpha$ and flux. From Figure 4 , it can be seen that there is an indication of hardening of the spectra as flux increases. We found $\rho=-0.5$ with $p=0.13$ between $\alpha$ versus flux with $\Delta \alpha=0.26$ during our observations. Hence, we conclude that there is no significant spectral variations with flux in the blazar, as also suggested by the HR analysis presented above. We also investigated the correlation between $\beta$ versus flux, which indicates the efficient stochastic acceleration of $X$-ray emitting electrons [34]. The correlation value $\rho=-0.35$ with $p=0.39$, hence we conclude that there is no significant correlation between them. Moreover, a positive correlation between $\alpha$ versus $\beta$ is expected within the energy dependent accerelation scenario. We found spearman correlation coefficient $\rho=-0.53$ with $p=0.14$. Hence, there is a weak negative correlation between them, which indicates that other types of accerelation mechanisms and cooling processes are at work. 
Table 4. Best spectral fit parameters for the Power Law, Log Parabolic and Broken Power Law Model for XMM-Newton Observations of Blazar H 2356-309.

\begin{tabular}{|c|c|c|c|c|c|c|c|c|}
\hline $\begin{array}{c}\text { Obs. Date } \\
\text { (yyyy-mm-dd) }\end{array}$ & $\Gamma_{1}$ & b or $\Gamma_{2}$ & $\begin{array}{c}E_{b} \\
(\mathrm{keV})\end{array}$ & $\begin{array}{l}\log _{10} \text { Flux } \\
\left(\mathrm{ergs} / \mathrm{s} / \mathrm{cm}^{2}\right)\end{array}$ & $\chi_{\text {Red }}^{2}$ & DoF & F-Test & $p$-Value \\
\hline 2005.06 .13 & $2.281_{-0.006}^{+0.006}$ & - & - & $-10.767_{-0.002}^{+0.002}$ & 1.043 & 151 & - & - \\
\hline 2005.06 .15 & $2.200_{-0.006}^{+0.006}$ & - & - & $-10.719_{-0.002}^{+0.002}$ & 0.945 & 153 & - & - \\
\hline 2007.06.02 & $2.161_{-0.002}^{+0.000}$ & - & - & $-10.602_{-0.001}^{+0.001}$ & 1.365 & 168 & - & - \\
\hline 2012.11.18 & $2.028_{-0.002}^{+0.002}$ & - & - & $-10.512_{-0.001}^{+0.001}$ & 1.492 & 167 & - & - \\
\hline 2013.12 .02 & $2.197_{-0.006}^{+0.006}$ & - & - & $-10.698_{-0.002}^{+0.002}$ & 1.154 & 152 & - & - \\
\hline 2013.12.03 & $2.199_{-0.003}^{+0.003}$ & - & - & $-10.691_{-0.001}^{+0.001}$ & 2.225 & 160 & - & - \\
\hline 2013.12 .10 & $2.283_{-0.003}^{+0.003}$ & - & - & $-10.766_{-0.001}^{+0.001}$ & 3.348 & 162 & - & - \\
\hline 2013.12.12 & $2.266_{-0.003}^{+0.003}$ & - & - & $-10.762_{-0.001}^{+0.001}$ & 2.848 & 163 & - & - \\
\hline \multirow[t]{2}{*}{ 2013.12.24 } & $2.182_{-0.002}^{+0.002}$ & - & - & $-10.664_{-0.001}^{+0.001}$ & 2.813 & 164 & - & - \\
\hline & & b & & & & & & \\
\hline 2005.06 .13 & $2.254_{-0.020}^{+0.020}$ & $0.032_{-0.022}^{+0.022}$ & - & $-10.769_{-0.002}^{+0.002}$ & 1.036 & 150 & 1.013 & 0.316 \\
\hline 2005.06.15 & $2.228_{-0.019}^{+0.019}$ & $-0.033_{-0.022}^{+0.022}$ & - & $-10.717_{-0.003}^{+0.003}$ & 0.936 & 152 & 1.461 & 0.229 \\
\hline 2007.06.02 & $2.179_{-0.007}^{+0.007}$ & $-0.021_{-0.008}^{+0.008}$ & - & $-10.600_{-0.001}^{+0.001}$ & 1.328 & 167 & 4.653 & 0.032 \\
\hline 2012.11.18* & $1.994_{-0.006}^{+0.006}$ & $0.035_{-0.006}^{+0.006}$ & - & $-10.514_{-0.001}^{+0.001}$ & 1.316 & 166 & 22.201 & $5.167 \times 10^{-6}$ \\
\hline 2013.12.02 * & $\begin{array}{l}2.070_{-0.019}^{+0.019} \\
\end{array}$ & $0.144_{-0.020}^{+0.020}$ & - & $-10.707_{-0.002}^{+0.001}$ & 0.815 & 151 & 62.809 & $4.645 \times 10^{-13}$ \\
\hline 2013.12.03* & $2.051_{-0.010}^{+0.010}$ & $0.160_{-0.011}^{+0.011}$ & - & $-10.700_{-0.001}^{+0.001}$ & 0.834 & 159 & 265.191 & $1.049 \times 10^{-35}$ \\
\hline 2013.12.10* & $2.118_{-0.009}^{+0.009}$ & $0.184_{-0.010}^{+0.011}$ & - & $-10.776_{-0.001}^{+0.001}$ & 1.048 & 161 & 353.34 & $1.871 \times 10^{-42}$ \\
\hline 2013.12.12* & $2.145_{-0.008}^{+0.008}$ & $0.136_{-0.009}^{+0.009}$ & - & $-10.769_{-0.001}^{+0.001}$ & 1.372 & 162 & 174.28 & $1.757 \times 10^{-27}$ \\
\hline \multirow[t]{2}{*}{2013.12 .24 * } & $2.063_{-0.008}^{+0.008}$ & $0.129_{-0.008}^{+0.008}$ & - & $-10.670_{-0.001}^{+0.001}$ & 1.225 & 163 & 211.301 & $3.112 \times 10^{-31}$ \\
\hline & & $\Gamma_{2}$ & & & & & & \\
\hline 2012.11.18 & $2.014_{-0.004}^{+0.004}$ & $2.056_{-0.008}^{+0.008}$ & $2.314_{-0.327}^{+0.327}$ & $-10.514_{-0.001}^{+0.001}$ & 1.326 & 165 & - & - \\
\hline 2013.12.02 & $2.143_{-0.011}^{+0.011}$ & $2.311_{-0.023}^{+0.023}$ & $2.056_{-0.197}^{+0.197}$ & $-10.707_{-0.002}^{+0.002}$ & 0.804 & 150 & - & - \\
\hline 2013.12.03 & $2.135_{-0.006}^{+0.006}$ & $2.322_{-0.010}^{+0.010}$ & $2.087_{-0.103}^{+0.103}$ & $-10.698_{-0.001}^{+0.001}$ & 0.786 & 158 & - & - \\
\hline 2013.12.10 & $2.210_{-0.005}^{+0.005}$ & $2.414_{-0.010}^{+0.010}$ & $1.967_{-0.074}^{+0.074}$ & $-10.775_{-0.001}^{+0.001}$ & 1.026 & 160 & - & - \\
\hline 2013.12.12 & $2.217_{-0.005}^{+0.005}$ & $2.372_{-0.011}^{+0.010}$ & $2.098_{-0.107}^{+0.107}$ & $-10.769_{-0.001}^{+0.001}$ & 1.484 & 161 & - & - \\
\hline 2013.12.24 & $2.130_{-0.005}^{+0.005}$ & $2.286_{-0.010}^{+0.010}$ & $2.137_{-0.100}^{+0.100}$ & $-10.670_{-0.001}^{+0.001}$ & 1.174 & 162 & - & - \\
\hline
\end{tabular}

$\Gamma_{1}$ : Low energy spectral index; $\Gamma_{2}$ : High energy spectral index; b: curvature; $E_{b}$ : Break Energy; $\chi_{\text {Red }}^{2}$ : Reduced $\chi^{2}$; DoF: degree of freedom; * indicates the observations that are well fitted by the LP model.
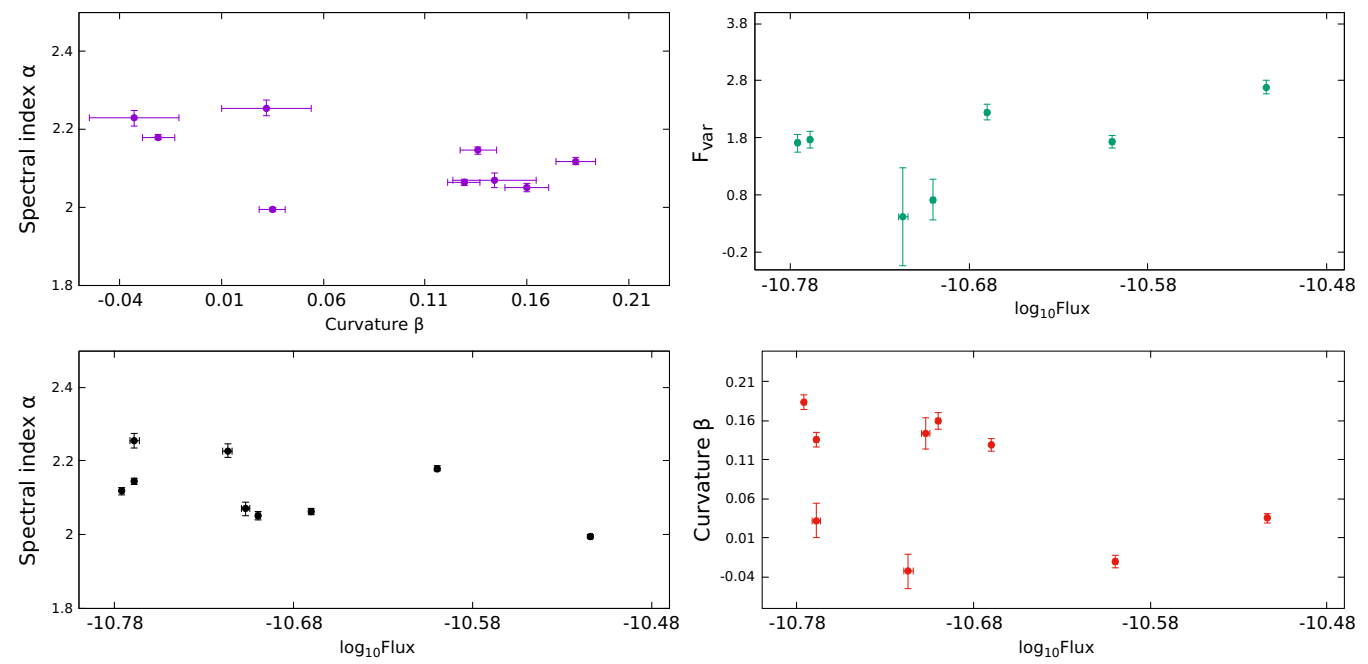

Figure 4. Relation between $\alpha$ versus $\beta$; $F_{\text {var }}$ versus Flux; $\alpha$ versus Flux and $\beta$ versus Flux.

\section{Discussion}

The study of X-ray flux variability on intra-day timescales is an important tool to understand the emission mechanism in blazars. It can be used to estimate the size and constrain the location and structure of a dominant emitting region (e.g., [39]). In blazars, most intrinsic flux variability across 
the EM bands can be explained by standard relativistic jet-based models (e.g., [2,40]), as it is closely aligned to our line of sight, while accretion-disk-based models are not very important for such sources, particularly when they are in high state. Accretion disk contribution is noticable in blazars, when they are observed in a low flux state $[39,40]$.

For HSP blazars, first hump of the SED peaks in UV / X-rays and, hence, they are brightest in X-ray bands. They show strong variability in this band, along with the large amplitude variations. In this work, we presented the timing and spectral analysis of nine observations of the HSP blazar H 2356-309 performed during 2005-2013. We used the fractional variability method to examine the intra-day variability in all of the light curves. For the HSP blazar H 2356-309 studied here, we found significant flux variability in five of the light curves out of total nine observations. Amplitude variations are between 1.71-2.69\% for these observations.

As we already mentioned that the accepted model for the strong variability in blazars on long term timescales involve shocks propagating down relativistic jets pointing close to our line of sight (i.e., [2,7]). The shorter variations or variations on IDV timescales can be mostly explained in terms of irregularities in the jet flows; turbulence behind the jet [40]; jets-in-a-jet model (i.e., [41]); magnetic reconnections taking place in fast-moving emitting regions within jets that accelerate the particles to high energies [42]. The shortest variability in the X-ray band (3-79 keV) is shown by the TeV blazar Mrk 421 with doubling time of $\sim 14$ minutes during its April 2013 flare [42].

We also examined the X-ray spectral variability of blazar H 2356-309 using the HR analysis. From the $\chi^{2}$ values in all of the observations, we found that there is no significant spectral variations in any of the observations. The fractional variability amplitude of TeV HSPs are studied in detail in literature and it is found that it ranges from few per cent to around 50\% during flaring events (e.g., [22,43,44]). Ref. [22] also studied the blazar H 2356-309 in 2018 using the NuSTAR observations, but did not find any significant flux or spectral variability.

It is well known that the spectra of high energy peaked blazars is intrinsically curved and, hence, log parabolic model better describes their spectra. The curved spectra are thought to originate from an energy dependent particle acceleration process ([34-36,45]). The curvature of the X-ray spectra can be either convex or concave. Convex curvature of the X-ray spectra, is likely to be caused by a single accelerated particle distribution (e.g., [34]). The concave X-ray spectra may be a consequence of the spectral upturn at the interaction of the high-energy tail of the synchrotron emission and the low-energy part of the inverse Compton emission (e.g., [46,47]). In order to study the X-ray spectral shape of blazar H 2356-309, we fit all of our observations using the power law and log parabolic model. We found that only three of our observations are well fitted by the power law model. Six of the observations showed spectral curvature and are well fitted by log parabolic model. Their photon indices varies between 1.99-2.15 and curvature values varies between 0.03-0.18.

In the energy dependent acceleration mechanism, a positive correlation is expected between $\alpha$ and $\beta$ ([34,35]). However, in our observations of the blazar H 2356-309, we found a weak negative correlation between them. Additionally, we searched for correlation between $\alpha$ versus flux in order to search for spectral variability but no significant variation is found between them. These results are in agreement with the studies of HR analysis. No correlation is found between $\beta$ versus flux and amplitude of variability versus flux.

\section{Conclusions}

We studied nine XMM-Newton observations of the HSP blazar H 2356-309, which are available in its public archive. Our conclusions are summarized, as follows:

1. The fractional variability analysis shows that there is moderate amplitude IDV for five of the total nine observations. Flux variability is observed in soft band $(0.3-2 \mathrm{keV})$ in all of these $5 \mathrm{LCs}$, but only three light curves showed flux variability in hard band (2-10 keV).

2. The variability amplitude is lower in the soft bands than in the hard bands that can be incorporated in energy dependent synchrotron model. We also estimated the IDV timescale for 
all nine observation IDs in $0.3-2 \mathrm{keV}$ (soft band) and 2-10 keV (hard band), but did not find any significant variability timescales.

3. HR analysis of all the nine observations shows that there is no significant spectral variability associated with the flux variability.

4. We fit all the spectra with power law and log parabolic model. Six of the observations are well fitted by log parabolic model indicating curvature ranging between $0.03-0.18$ and alpha varies between 1.99-2.15. Remaining three observations are well fitted by the power law model. The break energy is constrained to be $\sim 2.1 \mathrm{keV}$.

5. We searched for the correlations between $\alpha$ versus $\beta$, but found very weak negative correlation between them. We did not find any significant correlation between $\beta$ versus flux. No significant correlation between $\alpha$ versus flux is found, which again indicates no significant spectral variations in this source.

Author Contributions: K.A.W. analyzed the X-ray data, carried out timing and spectral analysis and wrote the initial manuscript. H.G. conceived the idea and finalize the manuscript. All authors have read and agreed to the published version of the manuscript.

Funding: This research is based on observations obtained with XMM-Newton, an ESA science mission with instruments and contributions directly funded by ESA member states and NASA.

Acknowledgments: Authors would like to thank the referees for their useful comments. Authors acknowledge the financial support from the Department of Science and Technology, India, through INSPIRE faculty award IFA17-PH197 at ARIES, Nainital.

Conflicts of Interest: The authors declare no conflict of interest.

\section{References}

1. Urry, C.M.; Padovani, P. Unified Schemes for Radio-Loud Active Galactic Nuclei. Publ. Astron. Soc. Pac. 1995, 107, 803. [CrossRef]

2. Marscher, A.P.; Gear, W.K. Models for high-frequency radio outbursts in extragalactic sources, with application to the early 1983 millimeter-to-infrared flare of 3C 273. Astrophys. J. 1985, 298, 114-127. [CrossRef]

3. Sikora, M.; Begelman, M.C.; Rees, M.J. Comptonization of Diffuse Ambient Radiation by a Relativistic Jet: The Source of Gamma Rays from Blazars? Astrophys. J. 1994, 421, 153. [CrossRef]

4. Padovani, P.; Giommi, P. The Connection between X-ray- and Radio-selected BL Lacertae Objects. Astrophys. J. 1995, 444, 567. [CrossRef]

5. Abdo, A.A.; Ackermann, M.; Ajello, M.; Axelsson, M.; Baldini, L.; Ballet, J.; Barbiellini, G.; Bastieri, D.; Baughman, B.M.; Bechtol, K.; et al. The Spectral Energy Distribution of Fermi Bright Blazars. Astrophys. J. 2010, 716, 30. [CrossRef]

6. Ulrich, M.-H.; Maraschi, L.; Urry, C.M. Variability of active galactic nuclei. Ann. Rev. Astron. Astrophys. 1997, 35, 445. [CrossRef]

7. Wagner, S.J.; Witzel, A. Intraday Variability in Quasars and BL Lac Objects. Ann. Rev. Astron. Astrophys. 1995, 33, 163. [CrossRef]

8. Gupta, A.C.; Banerjee, D.P.K.; Ashok, N.M.; Joshi, U.C. Near Infrared Intraday Variability of Mrk 421. Astron. Astrophys. 2004, 422, 505. [CrossRef]

9. Sembay, S.; Warwick, R.S.; Urry, C.M.; Sokoloski, J.; George, I.M.; Makino, F.; Ohashi, T.; Tashiro, M. The X-ray spectral variability of the BL Lacertae type object PKS 2155-304. Astrophys. J. 1993, 404, 112. [CrossRef]

10. Brinkmann, W.; Papadakis, I.E.; Raeth, C.; Mimica, P.; Haberl, F. XMM-Newton timing mode observations of Mrk 421. Astron. Astrophys. 2005, 443, 397-411. [CrossRef]

11. Zhang, Y.H.; Treves, A.; Celotti, A.; Qin, Y.P.; Bai, J.M. XMM-Newton View of PKS 2155-304: Characterizing the X-ray Variability Properties with EPIC pn. Astrophys. J. 2005, 629, 686-699. [CrossRef]

12. Zhang, Y.H. XMM-Newton Observations of the TeV BL Lacertae Object PKS 2155-304 in 2006: Signature of Inverse Compton X-ray Emission? Astrophys. J. 2008, 682, 789-797. [CrossRef]

13. Gaur, H.; Gupta, A.C.; Lachowicz, P.; Wiita, P.J. Detection of Intra-day Variability Timescales of Four High-Energy Peaked Blazars with XMM-Newton. Astrophys. J. 2010, 718, 279-291. [CrossRef] 
14. Kapanadze, B.; Romano, P.; Vercellone, S.; Kapanadze, S. The X-ray behaviour of the high-energy peaked BL Lacertae source PKS 2155-304 in the 0.3-10 keV band. Mon. Not. R. Astron. Soc. 2014, 444, 1077-1094. [CrossRef]

15. Schwartz, D.; Brissenden, R.J.V.; Thuoy, I.R. Lecture Notes in Physics; Maraschi, L., Maccacaro, T., Ulrich, H., Eds.; Springer: Berlin, Germany, 1989; Volume 334, p. 211.

16. Falomo, R. On the Galaxy Surrounding the BL Lac Object H2356-309. Astron. J. 1991, 101, 821. [CrossRef]

17. Costamante, L.; Ghisellini, G.; Giommi, P.; Tagliaferri, G.; Celotti, A.; Chiaberge, M.; Chiappetti, L.; Fossati, G.; Maraschi, L.; Pian, E.; et al. New Extreme Synchrotron BL Lac Objects. AIP Conf. Proc. 2001, 599, 586-589.

18. Giommi, P.; Colafrancesco, S. Non-thermal cosmic backgrounds and prospects for future high-energy observations of blazars. Exp. Astron. 2005, 20, 31-40. [CrossRef]

19. Aharonian, F.; Akhperjanian, A.G.; Bazer-Bachi, A.R.; Beilicke, M.; Benbow, W.; Berge, D.; Bernlohr, K.; Boisson, C.; Bolz, O.; Borrel, V.; et al. Discovery of very high energy $\gamma$-ray emission from the BL Lacertae object H 2356-309 with the HESS Cherenkov telescopes. Astron. Astrophys. 2006, 455, 461-466. [CrossRef]

20. Forman, W.; Jones, C.; Cominsky, L. The fourth Uhuru catalog of X-ray sources. Astrophys. J. Suppl. Ser. 1978, 38, 357. [CrossRef]

21. Wood, K.S.; Meekins, J.F.; Yentis, D.J. The HEAO A-1 X-ray source catalog. Astrophys. J. Suppl. Ser. 1984, 56, 507. [CrossRef]

22. Pandey, A.; Gupta, A.C.; Wiita, P.J. X-ray Flux and Spectral Variability of Six TeV Blazars with NuSTAR. Astrophys. J. 2018, 859, 49. [CrossRef]

23. Fang, T.; Danforth, C.; Buote, D.A.; Stocke, J.; Shull, M.; Canizares, C.R.; Gastaldello, F. An HST/COS Observation of Broad Ly $\alpha$ Emission and Associated Absorption Lines of the BL Lacertae Object H 2356-309. Astrophys. J. 2014, 795, 57. [CrossRef]

24. Abramowski, A.; et al. [HESS Collaboration]. Multi-wavelength Observations of H 2356-309. Astron. Astrophys. 2010, 516, A56. [CrossRef]

25. Jansen, F. XMM-Newton observatory I. The spacecraft and operations. Astron. Astrophys. 2001, 365, L1-L6. [CrossRef]

26. Nandra, K.; George, I.M.; Mushotzky, R.F.; Turner, T.J.; Yaqoob, T. ASCA Observations of Seyfert 1 Galaxies I. Data Analysis, Imaging And Timing. Astrophys. J. 1997, 476, 70. [CrossRef]

27. Edelson, R.; Turner, T.J.; Pounds, K.; Vaughan, S.; Markowitz, A.; Marshall, H.; Dobbie, P.; Warwick, R. X-ray Spectral Variability and Rapid Variability of the Soft X-ray Spectrum Seyfert 1 Galaxies Akn 564 and Ton S180. Astrophys. J. 2002, 568, 610. [CrossRef]

28. Edelson, R.; Pike, G.F.; Krolik, J.H. Broad-Band Properties of the CfA Seyfert Galaxies. III. Ultraviolet Variability. Astrophys. J. 1990, 359, 86. [CrossRef]

29. Rodriguez-Pascual, P.M.; Alloin, D.; Clavel, J.; Crenshaw, D.M.; Horne, K.; Kriss, G.A.; Krolik, J.H.; Malkan, M.A.; Netzer, H.; O'Brien, P.T.; et al. Steps Towards Determination of the Size and Structure of the Broad-Line Region in Active Galactic Nuclei. IX. Ultraviolet Observations of Fairall 9. Astrophys. J. Suppl. Ser. 1997, 110, 9. [CrossRef]

30. Vaughan, S.; Edelson, R.; Warwick, R.S.; Uttley, P. On characterising the variability properties of X-ray light curves from active galaxies. Mon. Not. R. Astron. Soc. 2003, 345, 1271. [CrossRef]

31. Jin, Y.K.; Zhang, S.N.; Wu, J.F. Hardness Ratio Estimation in Low Counting X-ray Photometry. Astrophys. J. 2006, 653, 1566-1570. [CrossRef]

32. Sivakoff, G.R.; Sarazin, C.L.; Carlin, J.L. Chandra Observations Of Diffuse Gas And Luminous X-ray Sources Around The X-ray-Bright Elliptical Galaxy NGC 1600. Astrophys. J. 2004, 617, 262. [CrossRef]

33. Lockman, J.; Savage, D. The Hubble Space Telescope Quasar Absorption Line Key Project. X. Galactic H i 21 Centimeter Emission toward 143 Quasars and Active Galactic Nuclei. Astrophys. J. Suppl. Ser. 1995, 97, 1-47. [CrossRef]

34. Massaro, E.; Perri, M.; Giommi, P.; Nesci, R. Log-parabolic spectra and particle acceleration in the BL Lac object Mkn 421: Spectral analysis of the complete BeppoSAX wide band X-ray data set. Astron. Astrophys. 2004, 413, 489. [CrossRef]

35. Massaro, F.; Tramacere, A.; Cavaliere, A.; Perri, M.; Giommi, P. X-ray spectral evolution of TeV BL Lacertae objects: eleven years of observations with BeppoSAX, XMM-Newton and Swift satellites. Astron. Astrophys. 2008, 478, 395. [CrossRef] 
36. Tramacere, A.; Massaro, F.; Cavaliere, A. Signatures of synchrotron emission and of electron acceleration in the X-ray spectra of Mrk 421. Astron. Astrophys. 2007, 466, 521-529. [CrossRef]

37. Tramacere, A.; Giommi, P.; Perri, M.; Verrecchia, F.; Tosti, G. Swift observations of the very intense flaring activity of Mrk 421 during 2006. I. Phenomenological picture of electron acceleration and predictions for $\mathrm{MeV} / \mathrm{GeV}$ emission. Astron. Astrophys. 2009, 501, 879-898. [CrossRef]

38. Landau, R.; Golisch, B.; Jones, T.J.; Jones, T.W.; Pedelty, J.; Rudnick, L.; Sitko, M.L.; Kenney, J.; Roellig, T.; Salonen, E.; et al. Active Extragalactic Sources: Nearly Simultaneous Observations from 20 Centimeters to 1400 Angstrom. Astrophys. J. 1986, 308, 78. [CrossRef]

39. Ciprini, S.; Tosti, G.; Raiteri, C.M.; Villata, M.; Ibrahimov, M.A.; Nucciarelli, G.; Lanteri, L. Optical variability of the BL Lacertae object GC 0109+224. Multiband behaviour and time scales from a 7-years monitoring campaign. Astron. Astrophys. 2003, 400, 487. [CrossRef]

40. Calafut, V.; Wiita, P.J. Modeling the Emission from Turbulent Relativistic Jets in Active Galactic Nuclei. J. Astrophys. Astron. 2015, 36, 255. [CrossRef]

41. Giannios, D.; Uzdensky, D.A.; Begelman, M.C. Fast TeV variability in blazars: jets in a jet. Mon. Not. R. Astron. Soc. 2009, 395, L29-L33. [CrossRef]

42. Paliya, V.S.; Böttcher, M.; Diltz, C.; Stalinl, C.S.; Sahayanathan, S.; Ravikumar, C.D. The violent hard X-ray variability of Mrk 421 observed by Nustar in 2013 April. Astrophys. J. 2015, 811, 143. [CrossRef]

43. Pandey, A.; Gupta, A.C.; Wiita, P.J. X-ray Intraday Variability of Five TeV Blazars with NuSTAR. Astrophys. J. 2017, 841, 123. [CrossRef]

44. Aggrawal, V.; Pandey, A.; Gupta, A.C.; Zhang, Z.; Wiita, P.J.; Yadav, K.K.; Tiwari, S.N. X-ray intraday variability of the TeV blazar Mrk 421 with Chandra. Mon. Not. R. Astron. Soc. 2018, 480, 4873-4883. [CrossRef]

45. Gaur, H.; Gupta, A.C.; Bachev, R.; Strigachev, A.; Semkov, E.; Wiita, P.J.; Gu, M.; Ibryamov, S. Multi-Band Intra-Night Optical Variability of BL Lacertae. Galaxies 2017, 5, 94. [CrossRef]

46. Gaur, H.; Mohan, P.; Wierzcholska, A.; Gu, M. Signature of inverse Compton emission from blazars. Mon. Not. R. Astron. Soc. 2018, 473, 3638-3660. [CrossRef]

47. Wierzcholska, A.; Wagner, S.J. X-ray spectral studies of $\mathrm{TeV} \gamma$-ray emitting blazars. Mon. Not. R. Astron. Soc. 2016, 458, 56-83. [CrossRef]

(C) 2020 by the authors. Licensee MDPI, Basel, Switzerland. This article is an open access article distributed under the terms and conditions of the Creative Commons Attribution (CC BY) license (http://creativecommons.org/licenses/by/4.0/). 